\title{
Estimation of general combining ability (GCA) in fifteen Cucumis sativus L inbred lines of the fifth generations
}

\author{
Hiet D. Hoang ${ }^{1 *},{\text { Phuong T. B. } \text { Nguyen }^{1} \text {, Trinh T. T. To }}^{1}$, Lam V. Tran ${ }^{1}$, Man T. T. Le ${ }^{1}$, \\ Tuan Q. Huynh ${ }^{1}$, Bich T. Thai ${ }^{1}$, \& Dan T. Vo ${ }^{2}$ \\ ${ }^{1}$ Research and Development Center for Hi-tech Agriculture, Ho Chi Minh City, Vietnam \\ ${ }^{2}$ Faculty of Agronomy, Nong Lam University, Ho Chi Minh City, Vietnam
}

ARTICLE INFO
Research Paper
Received: January 06, 2021
Revised: June 15, 2021
Accepted: June 23, 2021
Keywords
Cucumber
Cucumis sativus L.
General combining ability (GCA)
Yield

${ }^{*}$ Corresponding author

Hoang Dac Hiet

Email: hoanghiet.ahrd@gmail.com

\begin{abstract}
The study on General Combining ability (GCA) of 15 cucumber inbred lines $\left(\mathrm{I}_{5}\right)$ was carried out at the Research and Development Center for High-tech Agriculture, Ho Chi Minh City. The objective of the study was to identify the appropriate parental lines for producing F1 hybird cucumber cultivars. Experimental materials included 15 cucumber inbred lines ( $\left.\mathrm{I}_{5}\right)$ (L3, L9, L21, L22, L28, L32, L33, L36, L39, L61, L63, L69, L71, L74 and L78) and two test lines (T1 - TN 456 from Trang Nong Company, imported from Thailand and T2 Cuct 1450 from Chia Tai - Thailand), forming 30 hybrid combinations by the method of topcross. The results showed that seven inbred lines including L3, L9, L21, L28, L33, L61 and L71 had high ability to combine with both $\mathrm{T} 1$ and $\mathrm{T} 2$ tester lines ${ }^{\mathrm{ab}}$ by fruit yield with GCA index from $+(0.8)$ to $+(9.4)$.

Evaluation of 30 cucumber hybrid was combinations on the growth and fruit yield characteristics showed that L28/T1 and L71/T2 hybrids were significantly different from other hybrids with high yields of 43.1 tons/ha and 41.3 tons/ha, respectively.
\end{abstract}

Cited as: Hoang, H. D., Nguyen, P. T. B., To, T. T. T., Tran, L. V., Le, M. T. T., Huynh, T. Q., Thai, B. T., \& Vo, D. T. (2021). Estimation of general combining ability (GCA) in fifteen Cucumis sativus L inbred lines of the fifth generations. The Journal of Agriculture and Development 20(4), $1-9$. 


\title{
Đánh giá khả năng kết hợp chung của 15 dòng dưa leo (Cucumis sativus L.) thế hệ $\mathrm{I}_{5}$
}

\author{
Hoàng Đắc Hiệt ${ }^{1 *}$, Nguyễn Thị Bích Phượng ${ }^{1}$, Tô Thị Thùy Trinh ${ }^{1}$, Trần Văn Lâm ${ }^{1}$, \\ Lê Thị Thu Mận ${ }^{1}$, Huỳnh Quang Tuấn ${ }^{1}$, Thái Thị Bích ${ }^{1} \&$ Võ Thái Dân ${ }^{2}$ \\ ${ }^{1}$ Trung Tâm Nghiên Cứu và Phát Triển Nông Nghiệp Công Nghệ Cao, TP. Hồ Chí Minh \\ ${ }^{2}$ Khoa Nông Học, Trường Đại Học Nông Lâm TP.HCM, TP. Hồ Chí Minh
}

\section{THÔNG TIN BÀI BÁO}

Bài báo khoa học

Ngày nhận: 06/01/2021

Ngày chỉnh sửa: $15 / 06 / 2021$

Ngày chấp nhận: 23/06/2021

\section{Từ khóa}

Cây dưa leo

Cucumis sativus L.

Khả năng kết hợp chung

Năng suất

*Tác giả liên hệ

Hoàng Đắc Hiệt

Email: hoanghiet.ahrd@gmail.com

\section{TÓM TẮT}

Nghiên cứu đánh giá khả năng kết hợp chung của 15 dòng dưa leo $\mathrm{I}_{5}$ đã được thực hiện tại Trung tâm Nghiên cứu và Phát triển Nông nghiệp Công nghệ cao Thành phố Hồ Chí Minh. Mục tiêu của nghiên cứu nhằm xác định dòng bố mẹ phục vụ công tác tạo giống dưa leo lai F1. Vật liệu thí nghiệm gồm có 15 dòng dưa leo thế hệ $\mathrm{I}_{5}$ làm mẹ (L3, L9, L21, L22, L28, L32, L33, L36, L39, L61, L63, L69, L71, L74 và $\mathrm{L} 78$ ) và 2 dòng thử tester $\mathrm{T} 1$ và $\mathrm{T} 2$ ( $\mathrm{T} 1$ là giống $\mathrm{TN} 456$ của Công ty Trang Nông nhập khẩu từ Thái Lan và T2 là giống Cuct 1450 của Công ty Chia Tai - Thái Lan), tạo thành 30 tổ hợp lai bằng phương pháp lai đỉnh.

Kết quả chọn được 7 dòng gồm L3, L9, L21, L28, L33, L61 và L71 có khả năng kết hợp chung cao với cả 2 dòng tester $\mathrm{T} 1$ và $\mathrm{T} 2$ về năng suất với chỉ số KNKHC từ + $(0,8)$ đến + $(9,4)$. Đánh giá 30 tổ hợp lai về các chỉ tiêu về sinh trưởng và năng suất chọn được 2 tổ hợp lai với năng suất cao có ý nghĩa khác biệt so với các tổ hợp lai khác là L28/T1 và L71/T2 có năng suất lần lượt là 43,1 tấn/ha và 41,3 tấn/ha.

\section{1. Đặt Vấn Đề}

Cây dưa leo (Cucumis sativus L.) là loại cây rau ăn quả có giá trị thương mại lớn, được trồng phổ biến làm thực phẩm thông dụng ở nhiều nước trên thế giới. Dưa leo có hàm lượng vitamin và chất khoáng cao nên được ưa chuộng là sản phẩm rau phổ biến đứng hàng thứ tư sau cà chua, bắp cải và hành tây (Tatlioglu, 1993). Diện tích trồng dưa leo trên thế giới năm 2018 là 1.984.518 ha, năng suất trung bình đạt 37,9 tấn/ha và sản lượng đạt 7.5219.440 tấn. Tại Việt Nam, ước tính diện tích trồng dưa leo khoảng 31.570 ha/năm và sản lượng đạt 577.218 tấn/năm (FAOSTAT, 2020). Trong thực tế sản xuất, giống dưa leo được đưa vào sử dụng chủ yếu là giống nhập nội với ưu điểm cho năng suất cao nhưng giá thành hạt giống cao và không chủ động được nguồn giống. Chính vì vậy, việc chọn tạo các giống dưa leo lai F1 ở trong nước có năng suất và chất lượng cao hoặc tương đương với giống nhập nội nhưng giá thành hạt giống thấp sẽ góp phần chủ động trong việc cung cấp hạt giống cho người sản xuất và cải thiện lợi ích kinh tế cho người dân.

Trung tâm Nghiên cứu và Phát triển Nông nghiệp Công nghệ cao đã thu thập giống dưa leo địa phương và giống nhập nội trồng phổ biến tại các tỉnh miền Đông Nam Bộ từ năm 2017 dến 2019. Kết quả chọn được 15 dòng dưa leo thế hệ $\mathrm{I}_{5}$ phù hợp làm nguồn vật liệu để chọn tạo giống mới (Hoang \& ctv., 2019). Lai đỉnh có ý nghĩa đánh giá sớm để chọn các dòng tốt, đồng thời loại bỏ các dòng xấu nhằm tiết kiệm thời gian, nhân lực và phương pháp thí nghiệm (Hoang \& Truong, 2008). Vì vậy, nghiên cứu "Đánh giá khả năng kết hợp chung của 15 dòng dưa leo $(\mathrm{Cu}$ - 
cumis sativus L.) thế hệ $\mathrm{I}_{5}$ " đã được thực hiện. Nhằm mục tiêu đánh giá đặc điểm nông sinh học của các tổ hợp lai dưa leo, xác định khả năng kết hợp của các dòng bố mẹ theo các tính trạng năng suất, chọn lọc những tổ hợp lai có khả năng sinh trưởng tốt, năng suất cao và chống chịu sâu bệnh.

\section{Vật Liệu và Phương Pháp Nghiên Cứu}

\subsection{Vật liệu}

Giống gồm 30 tổ hợp lai được tạo thành bằng phương pháp lai đỉnh của 15 dòng dưa leo thế hệ

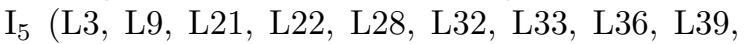
L61, L63, L69, L71, L74 và L78), kết quả các dòng dưa leo được kế thừa từ nghiên cứu chọn lọc nguồn vật liệu lai tạo giống tại Trung tâm Nghiên cứu và Phát triển Nông nghiệp Công nghệ cao trong thời gian từ năm 2017 - 2019. Hai dòng tester gồm T1 là giống TN 456 (Công ty Trang Nông nhập khẩu từ Thái Lan) và $\mathrm{T} 2$ là giống Cuct 1450 (Công ty Chia Tai - Thái Lan) (Bảng $1)$.

Thành phần giá thể trồng cây dưa leo gồm $70 \%$ mụn dừa và $30 \%$ phân trùn quế, phối trộn theo thể tích bầu trồng. Mụn dừa được xử lý sạch bằng cách ngâm xả nước trong 7 - 10 ngày, các chỉ số của mụn dừa đạt để đưa vào trồng cây như sau độ pH: 5,5; Lignin: 62 - 75\%; Tanin: 8,0 - 8,8\%; EC: $0,07 \mathrm{dS} / \mathrm{m}$. Phân trùn quế gồm chất hữu cơ (\%): C: 7,64 ; mùn: 13,17 ; chất tổng số $(\%)$ : N: 1,93: P: 0,71; K: 0,70 (AHRD, 2015).

Các loại hóa chất pha dung dịch thủy canh trồng dưa leo Monopotassium phosphate $\left[\mathrm{KH}_{2} \mathrm{PO}_{4}\right](23 \% \mathrm{P}$ và $28 \% \mathrm{~K}$; Jordan $)$, Calcium nitrate $\left[\mathrm{Ca}\left(\mathrm{NO}_{3}\right)_{2} \bullet 4 \mathrm{H}_{2} \mathrm{O}\right](16 \% \mathrm{~N}$ và $20 \%$ $\mathrm{Ca}$; Jordan), Potassium sulfate $\left[\mathrm{K}_{2} \mathrm{SO}_{4}\right](43,3 \%$ $\mathrm{K}$; Jordan), Magnesium sulfate $\left[\mathrm{MgSO}_{4} \bullet 7 \mathrm{H}_{2} \mathrm{O}\right]$ (11\% Mg; Jordan), Solubor $\left[\mathrm{H}_{3} \mathrm{BO}_{3}\right](20,5 \%$ $\mathrm{B}$; Ân Độ), Zinc sulfate $\left[\mathrm{ZnSO}_{4}\right]$ (36\% Zn; Ân Độ), Copper sulfate $\left[\mathrm{CuSO}_{4} .5 \mathrm{H}_{2} \mathrm{O}\right](25 \% \mathrm{Cu}$; Ấn Độ), Sodium molybdate $\left[\mathrm{Na}_{2} \mathrm{MoH}_{2} \mathrm{O}\right](39,6 \%$ Mo; Đức) và Chelated sắt ( $11 \% \mathrm{Fe}$; Ân Độ). Hóa chất sử dụng trong thí nghiệm có độ tinh khiết 89\% (AHRD, 2016).

\section{2. Điều kiện thí nghiệm}

Thí nghiệm được thực hiện trong điều kiện nhà màng kiểu máy thông gió cố định và hệ thống tưới nhỏ giọt theo thiết kế của Trung tâm Nghiên cứu và Phát triển Nông nghiệp Công nghệ cao.
Thời gian thí nghiệm từ tháng 08/2019 - 11/2019. Nhiệt độ trung bình trong nhà màng từ $32,8^{\circ} \mathrm{C}$ $36,9^{\circ} \mathrm{C}$, ẩm độ trung bình khoảng $53,9 \%$ - 67,7\%. Với nền nhiệt độ và ẩm độ này hoàn toàn thích hợp cho cây dưa leo sinh trưởng và phát triển bình thường trong nhà màng.

\subsection{Phương pháp nghiên cứu}

Thí nghiệm một yếu tố được bố trí kiểu hoàn toàn ngẫu nhiên (CRD) 30 nghiệm thức (gồm 30 tổ hợp lai) và 3 lần lặp lại. Mỗi ô thí nghiệm trồng 30 cây, khoảng cách trồng $(0,7 \times 0,5)$ x $1 \mathrm{~m}$. Tiến hành gieo ươm cây con trong khay xốp và trồng khi cây con có 2 lá thật, đạt 10 ngày sau gieo. Giá thể để trồng và ườm cây gồm $70 \%$ mụn dừa $+20 \%$ phân trùn quế và $10 \%$ tro trấu. Cây được trồng trong bầu với thể tích $5 \mathrm{dm}^{3}$ giá thể.

Các chỉ tiêu theo dõi: Các tính trạng về đặc điểm hình thái và mức độ nhiễm sâu bệnh hại được đánh giá theo quy chuẩn kỹ thuật quốc gia QCVN 01-87:2012/BNNPTNT về khảo nghiệm giá trị canh tác và sử dụng của giống dưa chuột (MARD, 2012). Thời gian ra hoa cái (ngày sau trồng) (Khi mỗi ô thí nghiệm có $50 \%$ số cây ra hoa cái), thời gian thu quả (ngày sau trồng) (Khi mỗi ô thí nghiệm có $50 \%$ số cây cho quả thu đợt đầu), chiều dài quả $(\mathrm{cm})$ (chọn 10 cây dưa leo ngẫu nhiên/ô, mỗi cây lâyy ngẫu nhiên 3 quả, đo khoảng cách giữa 2 đầu quả), đường kính quả $(\mathrm{cm})$ (chọn 10 cây dưa leo ngẫu nhiên/ô, mỗi cây lấy ngẫu nhiên 3 quả, đo phần đường kính lớn nhất trên quả), màu sắc quả (quan sát toàn ô màu xanh trắng, màu xanh nhạt, màu xanh trung bình và màu xanh đậm), hình dạng quả, gai quả, vị đắng ở đầu quả có cuống (đánh giá theo phương pháp cảm quan theo thang điểm từ 1 - 5: (1) Rất đắng; (2 - 4) Đắng; (5) Không đắng)), số quả trên thân chính (quả/thân chính), tổng số quả/cây (quả), khối lượng trung bình quả (g), năng suất cá thể (kg/cây), năng suất thực thu (tấn/ha), tỷ lệ (\%) bệnh sương mai (Pseudoperonospora sp.), bệnh phấn trắng (Eryshiphe sp.) và vi rút.

Cây dưa leo được chăm sóc theo quy trình kỹ thuật trồng dưa leo trên giá thể trong nhà màng. Giai đoạn cây từ trồng - ra hoa: 160 ppm N, 45 ppm P, 240 ppm K, ppm 130 Mg, 40 ppm Ca. Giai đoạn cây từ ra hoa đến hết vụ: 180 ppm N, 50 ppm P, 260 ppm K, ppm 150 ppm Mg, 50 ppm Ca. Nồng độ phân vi lượng bổ sung: 0,3 - 0,5 ppm B, 0,3 ppm Mn, 2 - 3 ppm Fe, 0,05 ppm Mo, 0,1 - 0,5 ppm Cu, 0,3 ppm Zn (AHRD, 2016). 
Bảng 1. Thời gian thu quả đầu tiên, khối lượng quả, chiều dài quả, đường kính quả và khối lượng quả/cây của 15 dòng dưa leo thế hệ $\mathrm{I}_{5}$ và 2 dòng thử $(\mathrm{T} 1, \mathrm{~T} 2)$

\begin{tabular}{ccccccc}
\hline Tên dòng & $\begin{array}{c}\text { Thời gian } \\
\text { thu quả } \\
\text { dầu tiên } \\
\text { (ngày) }\end{array}$ & $\begin{array}{c}\text { Khối lượng } \\
\text { quả } \\
(\mathrm{TB} \pm \mathrm{SE}) \\
(\mathrm{g})\end{array}$ & $\begin{array}{c}\text { Chiều dài } \\
\text { quả } \\
(\mathrm{TB} \pm \mathrm{SE}) \\
(\mathrm{cm})\end{array}$ & $\begin{array}{c}\text { Dường kính } \\
\text { quả } \\
(\mathrm{TB} \pm \mathrm{SE}) \\
(\mathrm{cm})\end{array}$ & $\begin{array}{c}\text { Độ dày } \\
\text { thịt quả } \\
(\mathrm{TB} \pm \mathrm{SE}) \\
(\mathrm{cm})\end{array}$ & $\begin{array}{c}\text { Khối lượng } \\
\text { quả/cây } \\
(\mathrm{TB} \pm \mathrm{SE}) \\
(\mathrm{kg})\end{array}$ \\
\hline L3.5 & 34 & $149,5 \pm 3,4$ & $19,3 \pm 0,5$ & $3,8 \pm 0,4$ & $1,4 \pm 0,2$ & $1,9 \pm 0,5$ \\
L9.5 & 33 & $145,4 \pm 3,4$ & $18,9 \pm 0,4$ & $3,7 \pm 0,3$ & $1,3 \pm 0,2$ & $1,8 \pm 0,6$ \\
L21.5 & 34 & $156,7 \pm 3,7$ & $19,1 \pm 0,6$ & $3,6 \pm 0,4$ & $1,3 \pm 0,3$ & $1,9 \pm 0,5$ \\
L22.5 & 34 & $144,9 \pm 3,7$ & $19,3 \pm 0,4$ & $3,5 \pm 0,4$ & $1,4 \pm 0,2$ & $1,8 \pm 0,6$ \\
L28.5 & 34 & $156,7 \pm 3,7$ & $19,2 \pm 0,4$ & $3,5 \pm 0,3$ & $1,3 \pm 0,3$ & $2,0 \pm 0,5$ \\
L32.5 & 33 & $156,4 \pm 3,4$ & $18,9 \pm 0,5$ & $3,6 \pm 0,3$ & $1,3 \pm 0,2$ & $1,9 \pm 0,6$ \\
L33.5 & 31 & $134,9 \pm 3,7$ & $19,4 \pm 0,4$ & $3,7 \pm 0,4$ & $1,4 \pm 0,3$ & $1,7 \pm 0,5$ \\
L36.5 & 32 & $146,4 \pm 3,9$ & $19,3 \pm 0,5$ & $3,5 \pm 0,3$ & $1,3 \pm 0,2$ & $1,8 \pm 0,5$ \\
L39.5 & 34 & $156,4 \pm 3,7$ & $19,3 \pm 0,5$ & $3,7 \pm 0,4$ & $1,4 \pm 0,2$ & $2,0 \pm 0,6$ \\
L61.5 & 33 & $167,7 \pm 3,7$ & $18,9 \pm 0,4$ & $3,9 \pm 0,3$ & $1,4 \pm 0,3$ & $2,1 \pm 0,6$ \\
L63.5 & 34 & $154,8 \pm 3,9$ & $19,4 \pm 0,5$ & $3,8 \pm 0,3$ & $1,3 \pm 0,2$ & $1,9 \pm 0,5$ \\
L69.5 & 33 & $155,6 \pm 3,4$ & $19,4 \pm 0,4$ & $3,7 \pm 0,4$ & $1,5 \pm 0,2$ & $1,9 \pm 0,5$ \\
L71.5 & 33 & $156,7 \pm 3,9$ & $19,4 \pm 0,5$ & $3,9 \pm 0,3$ & $1,4 \pm 0,3$ & $2,0 \pm 0,6$ \\
L74.5 & 34 & $153,9 \pm 3,7$ & $18,3 \pm 0,4$ & $3,7 \pm 0,4$ & $1,3 \pm 0,2$ & $1,9 \pm 0,5$ \\
L78.5 & 33 & $156,7 \pm 3,7$ & $19,3 \pm 0,6$ & $3,7 \pm 0,4$ & $1,4 \pm 0,3$ & $2,0 \pm 0,5$ \\
T1 & 35 & $158,1 \pm 3,2$ & $16,1 \pm 0,5$ & $3,9 \pm 0,3$ & $1,3 \pm 0,1$ & $2,0 \pm 0,4$ \\
T2 & 34 & $160,4 \pm 2.2$ & $18,3 \pm 0,2$ & $3,8 \pm 0,1$ & $1,4 \pm 0,3$ & $1,9 \pm 0,3$ \\
\hline
\end{tabular}

Nguồn: Hoang \& ctv. (2019).

\subsection{Phương pháp đánh giá khả năng kết hợp}

Đánh giá khả năng kết hợp chung yếu tố năng suất thực thu (Phan, 2006).

\subsection{Phương pháp xử lý số liệu}

Số liệu thí nghiệm được phân tích ANOVA, phân hạng trên phần mềm SAS 9.1; khả năng kết hợp chung được tính toán trên phần mềm Microsoft Excel.

\section{Kết Quả và Thảo Luận}

\subsection{Khả năng sinh trưởngcủa 30 tổ hợp lai từ 15 dòng dưa leo thế hệ $\mathrm{I}_{5}$}

Qua đánh giá sự sinh trưởng của 30 tổ hợp lai từ trồng đến bắt đầu ra hoa cái dao động từ 25 - 27 ngày sau trồng. Thời gian từ khi trồng đến thu quả đầu tiên dao động từ 32 - 36 ngày. Tổ hợp lai L28/T2 có thời gian từ khi trồng đến thu quả đầu tiên quả sớm nhất là 32 ngày. Tổ hợp lai L71/T1 có số quả trên thân chính nhiều nhất là 4,7 quả/thân và thấp nhất là 3,8 quả/thân. Các tổ hợp lai có tỷ lệ đậu quả trung bình dao động từ 58,2 đến $83,1 \%$.

\section{2. Đặc điểm các chỉ tiêu về quả của 30 tổ hợp lai từ 15 dòng dưa leo thế hệ $\mathrm{I}_{5}$}

Theo kết quả Bảng 2, các tổ hợp lai dưa leo có chiều dài quả trung bình từ 17,3 đến $19,7 \mathrm{~cm}$, đường kính quả là từ 3,3 đến $3,8 \mathrm{~cm}$. Các tổ hợp lai dưa leo đều có hình dạng quả là hình trụ và đều không có vị đắng ở đầu quả, màu sắc vỏ quả có 2 nhóm là màu xanh trung bình và xanh nhạt, gai quả có màu nâu xám và màu trắng. Theo Ngo (2011), giống có gai quả màu trắng sẽ cho thời gian thu hái dài hơn, đặc biệt là sản phẩm quả không bị biến vàng trong quá trình thu hoạch cũng như trong quá trình lưu kho trước khi chế biến. Giống dưa leo có gai quả màu nâu hoặc đen, quả dưa leo thường chuyển sang màu vàng sớm đẫn đến hiện tượng trái nhanh già. Đây là một trong những yếu tố chú ý trong quá trình chọn tạo giống. Kết quả này cũng tương đồng với Tran \& ctv. (2012), về tiêu chí chọn giống dưa leo có kích thước và màu sắc đáp ứng được thị hiếu người tiêu dùng. Chọn các tổ hợp lai giống dưa leo có triển vọng ở khu vực phía nam có chiều dài quả từ $15 \mathrm{~cm}$ đến $22 \mathrm{~cm}$, đường kính quả 3,8 đến 4,3 cm, vỏ quả có màu xanh hoặc màu xanh nhạt.

Hình dạng quả dưa leo của một số tổ hợp lai được chọn chủ yếu dạng hình trụ, quả thon dài, 
Bảng 2. Các đặc điểm về hình dạng quả, màu sắc vỏ quả, màu sắc gai quả và vị đắng ở đầu quả có cuống của các tổ hợp lai dưa leo thế hệ $\mathrm{I}_{5}$

\begin{tabular}{|c|c|c|c|c|c|c|}
\hline $\begin{array}{l}\text { Tổ hợp } \\
\text { lai }\end{array}$ & $\begin{array}{l}\text { Chiều dài } \\
\text { trung bình } \\
\text { quả }(\mathrm{cm})\end{array}$ & $\begin{array}{l}\text { Đường kính } \\
\text { trung bình } \\
\text { quả }(\mathrm{cm})\end{array}$ & $\begin{array}{c}\text { Hình dạng } \\
\text { quả }\end{array}$ & Màu sắc vỏ quả & $\begin{array}{l}\text { Màu sắc } \\
\text { gai quả }\end{array}$ & $\begin{array}{l}\text { Vị đắng ở } \\
\text { đầu quả } \\
\text { có cuống }\end{array}$ \\
\hline L3/T1 & 18,9 & 3,7 & Hình trụ & Xanh trung bình & Trắng & Không \\
\hline $\mathrm{L} 9 / \mathrm{T} 1$ & 19,5 & 3,5 & Hình trụ & Xanh trung bình & Trắng & Không \\
\hline $\mathrm{L} 21 / \mathrm{T} 1$ & 18,9 & 3,6 & Hình trụ & Xanh trung bình & Trắng & Không \\
\hline $\mathrm{L} 22 / \mathrm{T} 1$ & 17,9 & 3,5 & Hình trụ & Xanh nhạt & Trắng & Không \\
\hline $\mathrm{L} 28 / \mathrm{T} 1$ & 18,7 & 3,6 & Hình trụ & Xanh trung bình & Nâu xám & Không \\
\hline $\mathrm{L} 32 / \mathrm{T} 1$ & 18,0 & 3,6 & Hình trụ & Xanh trung bình & Trắng & Không \\
\hline $\mathrm{L} 33 / \mathrm{T} 1$ & 19,5 & 3,4 & Hình trụ & Xanh trung bình & Trắng & Không \\
\hline $\mathrm{L} 36 / \mathrm{T} 1$ & 17,9 & 3,5 & Hình trụ & Xanh trung bình & Trắng & Không \\
\hline L39/T1 & 18,6 & 3,4 & Hình trụ & Xanh trung bình & Trắng & Không \\
\hline $\mathrm{L} 61 / \mathrm{T} 1$ & 18,4 & 3,8 & Hình trụ & Xanh trung bình & Nâu xám & Không \\
\hline $\mathrm{L} 63 / \mathrm{T} 1$ & 18,7 & 3,9 & Hình trụ & Xanh trung bình & Nâu xám & Không \\
\hline L69/T1 & 18,1 & 3,8 & Hình trụ & Xanh trung bình & Nâu xám & Không \\
\hline $\mathrm{L} 71 / \mathrm{T} 1$ & 19,1 & 3,7 & Hình trụ & Xanh trung bình & Nâu xám & Không \\
\hline $\mathrm{L} 74 / \mathrm{T} 1$ & 18,7 & 3,6 & Hình trụ & Xanh trung bình & Nâu xám & Không \\
\hline $\mathrm{L} 78 / \mathrm{T} 1$ & 18,6 & 3,5 & Hình trụ & Xanh trung bình & Nâu xám & Không \\
\hline $\mathrm{L} 3 / \mathrm{T} 2$ & 18,7 & 3,6 & Hình trụ & Xanh nhạt & Trắng & Không \\
\hline $\mathrm{L} 9 / \mathrm{T} 2$ & 18,9 & 3,5 & Hình trụ & Xanh nhạt & Trắng & Không \\
\hline $\mathrm{L} 21 / \mathrm{T} 2$ & 18,6 & 3,6 & Hình trụ & Xanh nhạt & Trắng & Không \\
\hline $\mathrm{L} 22 / \mathrm{T} 2$ & 17,3 & 3,5 & Hình trụ & Xanh nhạt & Trắng & Không \\
\hline $\mathrm{L} 28 / \mathrm{T} 2$ & 18,6 & 3,7 & Hình trụ & Xanh nhạt & Trắng & Không \\
\hline $\mathrm{L} 32 / \mathrm{T} 2$ & 18,5 & 3,6 & Hình trụ & Xanh nhạt & Trắng & Không \\
\hline $\mathrm{L} 33 / \mathrm{T} 2$ & 17,6 & 3,3 & Hình trụ & Xanh trung bình & Nâu xám & Không \\
\hline $\mathrm{L} 36 / \mathrm{T} 2$ & 18,9 & 3,5 & Hình trụ & Xanh nhạt & Trắng & Không \\
\hline $\mathrm{L} 39 / \mathrm{T} 2$ & 18,2 & 3,4 & Hình trụ & Xanh nhạt & Trắng & Không \\
\hline $\mathrm{L} 61 / \mathrm{T} 2$ & 18,6 & 3,5 & Hình trụ & Xanh nhạt & Nâu xám & Không \\
\hline $\mathrm{L} 63 / \mathrm{T} 2$ & 18,9 & 3,6 & Hình trụ & Xanh nhạt & Nâu xám & Không \\
\hline $\mathrm{L} 69 / \mathrm{T} 2$ & 17,9 & 3,4 & Hình trụ & Xanh trung bình & Nâu xám & Không \\
\hline $\mathrm{L} 71 / \mathrm{T} 2$ & 19,2 & 3,6 & Hình trụ & Xanh nhạt & Trắng & Không \\
\hline $\mathrm{L} 74 / \mathrm{T} 2$ & 19,7 & 3,6 & Hình trụ & Xanh nhạt & Nâu xám & Không \\
\hline $\mathrm{L} 78 / \mathrm{T} 2$ & 17,9 & 3,4 & Hình trụ & Xanh nhạt & Nâu xám & Không \\
\hline CV (\%) & 5,7 & 7,4 & & & & \\
\hline $\mathrm{F}(\mathrm{t})$ & $0,9^{\mathrm{ns}}$ & $0,8^{\mathrm{ns}}$ & & & & \\
\hline
\end{tabular}

ns: không khác biệt.

đầu quả tròn. Mùa sắc quả dưa leo có màu xanh nhạt, có lớp phấn trắng bao phủ đều khắp quả (Hình $1,2,3,4,5$ và 6 ).

3.3. Các yếu tố cấu thành năng suất và khả năng kết hợp chung (KNKHC) của 30 tổ hợp lai giống dưa leo

Theo kết quả Bảng 3, về chỉ tiêu tổng số quả/cây của các tổ hợp lai dưa leo trung bình dao động từ 9,6 đến 14,1 quả/cây, tổ hợp lai L28/T1 có tổng số quả/cây là 14,1 không có sự khác biệt với tổ hợp lai L71/T2 nhưng khác biệt có rất có ý nghĩa với các tổ hợp lai khác trong thí nghiệm. Phân tích khả năng kết hợp chung về chỉ tiêu số quả trung bình trên cây đối với vật liệu thử $\mathrm{T} 1$ và $\mathrm{T} 2$, có 9 dòng có khả năng kết hợp chung cao, giá trị KNKHC từ $(+)$ 0,0 đến $(+) 2,6$ và 6 dòng không có khả năng kết hợp chung, giá trị KNKHC âm (-).

Về chỉ tiêu khối lượng quả, các tổ hợp lai dưa leo có khối lượng quả trong khoản từ 125,9 đến $167,4 \mathrm{~g} / q u a ̉$. Khả năng kết hợp chung về khối lượng quả với vật liệu thử T1 và T2, có 7 dòng 


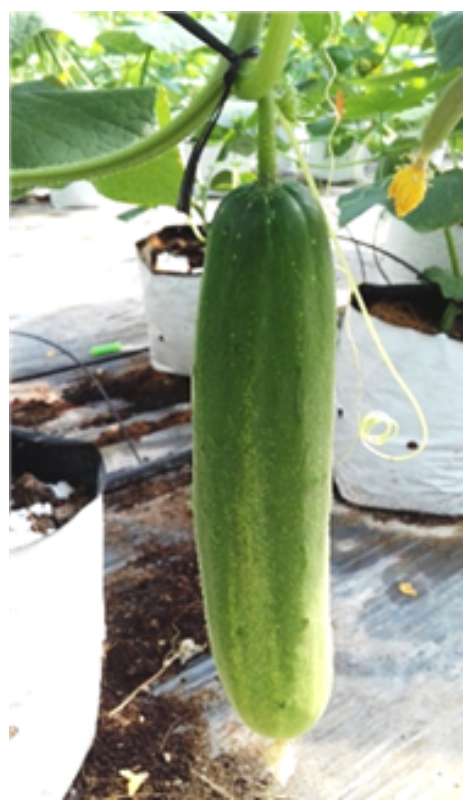

Hình 1. Hình dạng quả của tổ hợp lai L3/T1.

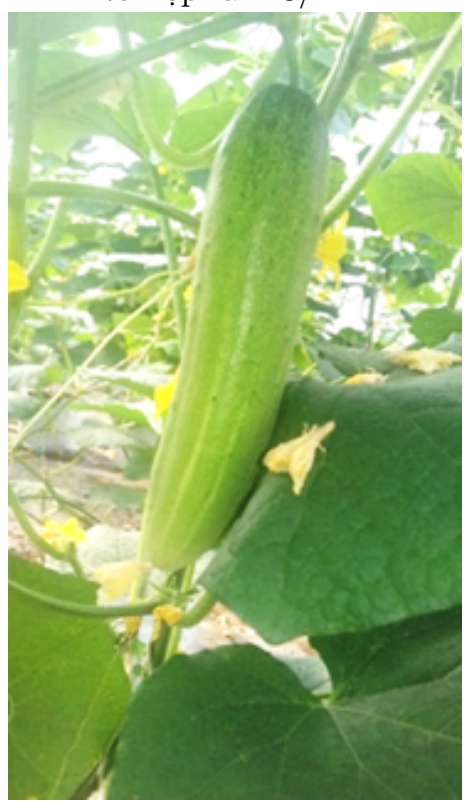

Hình 4. Hình dạng quả của tổ hợp lai L28/T1.

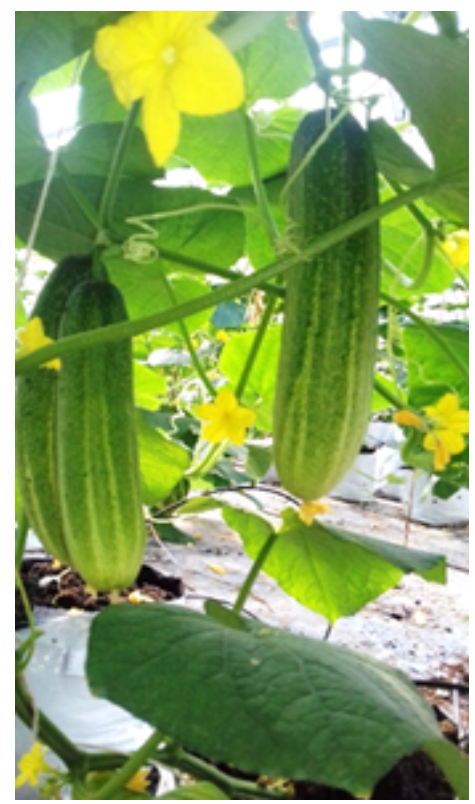

Hình 2. Hình dạng quả của tổ hợp lai L9/T1.

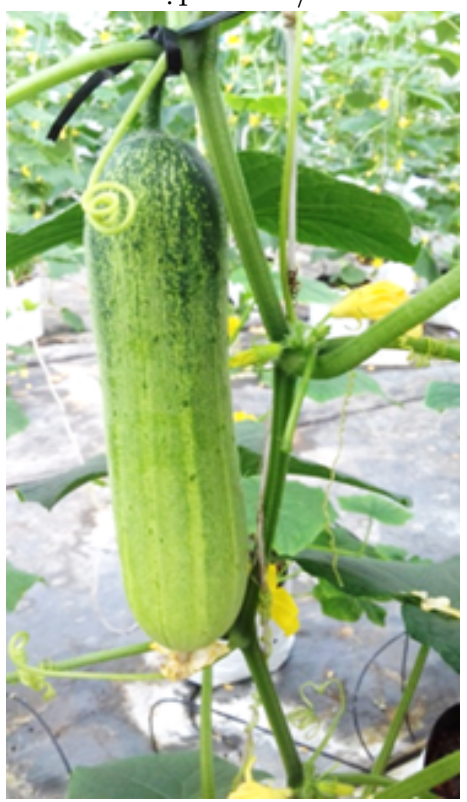

Hình 5. Hình dạng quả của tổ hợp lai L36/T1.

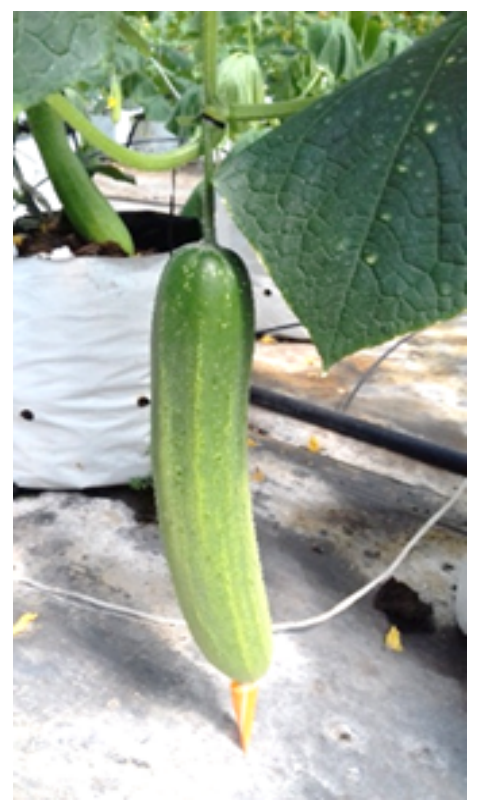

Hình 3. Hình dạng quả của tổ hợp lai L21/T1.

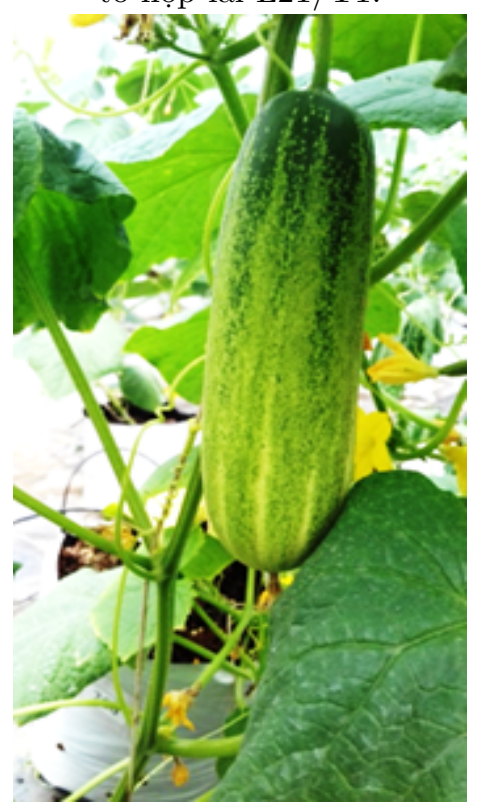

Hình 6. Hình dạng quả của tổ hợp lai L61/T1. có khả năng phối hợp chung cao, giá trị KNKHC từ $(+)$ 1,5 đến $(+) 15,6$ và 8 dòng không có khả năng kết hợp chung, giá trị KNKHC âm (-).

Về chỉ tiêu khối lượng quả/cây của các tổ hợp lai dưa leo dao động từ 1,3 đến $2,2 \mathrm{~kg} /$ cây. Tổ hợp lai L28/T1 có khối lượng quả/cây cao nhất $2,2 \mathrm{~kg} /$ cây không có sự khác biệt với các tổ hợp lai L3/T1, L9/T1, L21/T1, L33/T1, L61/T1,
L3/T2, L9/T2, L21/T2, L28/T2, L32/T2, $\mathrm{L} 36 / \mathrm{T} 2, \mathrm{~L} 61 / \mathrm{T} 2, \mathrm{~L} 63 / \mathrm{T} 2$ và $\mathrm{L} 71 / \mathrm{T} 2.7$ dòng dưa leo thế hệ I5 có khả năng kết hợp cao với tester T1 có giá trị chênh lệch $(+)$ 0,2 - (+) 0,5 gồm L3, L9, L21, L28, L33, L61, L71 và 10 dòng dưa leo có khả năng kết hợp cao với tester T1 có giá trị chênh lệch từ $(+)$ 0,0 - (+) 0,3 gồm L3, L9, L21, L28, L32, L36, L61, L63, L71, L74 (Bảng 3). 
Bảng 3. Các yếu tố cấu thành năng suất và khả năng kết hợp chung (KNKHC) của 30 tổ hợp lai giống dưa leo

\begin{tabular}{|c|c|c|c|c|c|c|}
\hline $\begin{array}{l}\text { Tổ hợp } \\
\text { lai }\end{array}$ & $\begin{array}{c}\text { Số quả } \\
\text { TB/cây } \\
\text { (quả/cây) }\end{array}$ & $\begin{array}{c}\text { KNKHC về } \\
\text { số quả } \\
\text { TB/cây }\end{array}$ & $\begin{array}{l}\text { Khối lượng } \\
\text { quả (g/quả) }\end{array}$ & $\begin{array}{l}\text { KNKHC về khối } \\
\text { lượng quả }\end{array}$ & $\begin{array}{c}\text { Khối } \\
\text { lượng } \\
\text { quả/cây } \\
\text { (kg/cây) }\end{array}$ & $\begin{array}{c}\text { KNKHC } \\
\text { về khối } \\
\text { lượng } \\
\text { quả/cây }\end{array}$ \\
\hline$\overline{\mathrm{L} 3 / \mathrm{T} 1}$ & $12,5^{\mathrm{c}}$ & 1,0 & $165,7^{\mathrm{a}}$ & 15,6 & $2,1^{\mathrm{a}}$ & 0,3 \\
\hline L9/T1 & $11,9^{\mathrm{c}-\mathrm{f}}$ & 0,4 & $164,8^{\mathrm{ab}}$ & 14,7 & $2,0^{\mathrm{ab}}$ & 0,2 \\
\hline L21/T1 & $12,8^{\mathrm{bc}}$ & 1,3 & $164,6^{\mathrm{ab}}$ & 14,5 & $2,1^{\mathrm{a}}$ & 0,4 \\
\hline $\mathrm{L} 22 / \mathrm{T} 1$ & $11,1^{\mathrm{efg}}$ & $-0,4$ & $150,1^{\mathrm{bcd}}$ & 0,0 & $1,7^{\mathrm{bcd}}$ & $-0,1$ \\
\hline $\mathrm{L} 28 / \mathrm{T} 1$ & $14,1^{\mathrm{a}}$ & 2,6 & $158,3^{\mathrm{a}-\mathrm{d}}$ & 8,2 & $2,2^{\mathrm{a}}$ & 0,5 \\
\hline L32/T1 & $10,9^{\mathrm{fgh}}$ & $-0,6$ & $124,8^{f}$ & $-25,3$ & $1,4^{\mathrm{de}}$ & $-0,4$ \\
\hline $\mathrm{L} 33 / \mathrm{T} 1$ & $12,5^{\mathrm{c}}$ & 1,0 & $157,4^{\mathrm{a}-\mathrm{d}}$ & 7,3 & $2,0^{\mathrm{ab}}$ & 0,2 \\
\hline L36/T1 & $9,6 \mathrm{i}$ & $-1,9$ & $149,8^{\mathrm{b}-\mathrm{e}}$ & $-0,3$ & $1,4^{\mathrm{de}}$ & $-0,3$ \\
\hline L39/T1 & $9,9^{\text {hi }}$ & $-1,6$ & $138,5^{\mathrm{ef}}$ & $-11,6$ & $1,4^{\mathrm{de}}$ & $-0,4$ \\
\hline $\mathrm{L} 61 / \mathrm{T} 1$ & $12,7^{\mathrm{bc}}$ & 1,2 & $165,7^{\mathrm{a}}$ & 15,6 & $2,1^{\mathrm{a}}$ & 0,4 \\
\hline L63/T1 & $10,7^{\mathrm{ghi}}$ & $-0,8$ & $125,9^{\mathrm{f}}$ & $-24,2$ & $1,3^{\mathrm{e}}$ & $-0,4$ \\
\hline L69/T1 & $9,6^{\mathrm{i}}$ & $-1,9$ & $143,4^{\mathrm{de}}$ & $-6,7$ & $1,4^{\mathrm{de}}$ & $-0,4$ \\
\hline $\mathrm{L} 71 / \mathrm{T} 1$ & $12,5^{\mathrm{c}}$ & 1,0 & $154,6^{\mathrm{a}-\mathrm{d}}$ & 4,5 & $1,9^{\mathrm{abc}}$ & 0,2 \\
\hline $\mathrm{L} 74 / \mathrm{T} 1$ & $10,9^{\text {fgh }}$ & $-0,6$ & $143,7^{\mathrm{de}}$ & $-6,4$ & $1,6^{\text {cde }}$ & $-0,2$ \\
\hline L78/T1 & $10,8^{\text {fgh }}$ & $-0,7$ & $144,7^{\text {cde }}$ & $-5,4$ & $1,6^{\mathrm{cde}}$ & $-0,2$ \\
\hline $\mathrm{L} 3 / \mathrm{T} 2$ & $11,9^{\mathrm{c}-\mathrm{f}}$ & 0,0 & $165,4^{\mathrm{a}}$ & 12,5 & $2,0^{\mathrm{ab}}$ & 0,1 \\
\hline L9/T2 & $12,1^{\text {cde }}$ & 0,2 & $154,4^{\mathrm{a}-\mathrm{d}}$ & 1,5 & $1,9^{\mathrm{abc}}$ & 0,0 \\
\hline $\mathrm{L} 21 / \mathrm{T} 2$ & $12,4^{\mathrm{c}}$ & 0,5 & $155,4^{\mathrm{a}-\mathrm{d}}$ & 2,5 & $1,9^{\mathrm{abc}}$ & 0,1 \\
\hline $\mathrm{L} 22 / \mathrm{T} 2$ & $10,9^{\text {fgh }}$ & $-1,0$ & $135,7^{\mathrm{ef}}$ & $-17,2$ & $1,5^{\mathrm{de}}$ & $-0,3$ \\
\hline $\mathrm{L} 28 / \mathrm{T} 2$ & $11,9^{\mathrm{c}-\mathrm{f}}$ & 0,0 & $158,4^{\mathrm{a}-\mathrm{d}}$ & 5,5 & $1,9^{\mathrm{abc}}$ & 0,1 \\
\hline L32/T2 & $12,8^{\mathrm{bc}}$ & 0,9 & $155,4^{\mathrm{a}-\mathrm{d}}$ & 2,5 & $2,0^{\mathrm{ab}}$ & 0,2 \\
\hline $\mathrm{L} 33 / \mathrm{T} 2$ & $11,2^{\mathrm{d}-\mathrm{g}}$ & $-0,7$ & $124,8^{f}$ & $-28,1$ & $1,4^{\mathrm{de}}$ & $-0,4$ \\
\hline $\mathrm{L} 36 / \mathrm{T} 2$ & $12,7^{\mathrm{bc}}$ & 0,8 & $159,7^{\mathrm{abc}}$ & 6,8 & $2,0^{\mathrm{ab}}$ & 0,2 \\
\hline $\mathrm{L} 39 / \mathrm{T} 2$ & $9,6^{\mathrm{i}}$ & $-2,3$ & $157,1^{\mathrm{a}-\mathrm{d}}$ & 4,2 & $1,5^{\mathrm{de}}$ & $-0,3$ \\
\hline $\mathrm{L} 61 / \mathrm{T} 2$ & $12,9^{\mathrm{bc}}$ & 1,0 & $155,3^{\mathrm{a}-\mathrm{d}}$ & 2,4 & $2,0^{\mathrm{ab}}$ & 0,2 \\
\hline $\mathrm{L} 63 / \mathrm{T} 2$ & $12,3^{\mathrm{cd}}$ & 0,4 & $167,4^{\mathrm{a}}$ & 14,5 & $2,1^{\mathrm{a}}$ & 0,2 \\
\hline L69/T2 & $10,7^{\mathrm{ghi}}$ & $-1,2$ & $144,2^{\mathrm{de}}$ & $-8,7$ & $1,5^{\mathrm{de}}$ & $-0,3$ \\
\hline $\mathrm{L} 71 / \mathrm{T} 2$ & $13,8^{\mathrm{ab}}$ & 1,9 & $154,8^{\mathrm{a}-\mathrm{d}}$ & 1,9 & $2,1^{\mathrm{a}}$ & 0,3 \\
\hline $\mathrm{L} 74 / \mathrm{T} 2$ & $12,9^{\mathrm{bc}}$ & 1,0 & $157,1^{\mathrm{a}-\mathrm{d}}$ & 4,2 & $2,0^{\mathrm{ab}}$ & 0,2 \\
\hline $\mathrm{L} 78 / \mathrm{T} 2$ & $10,7^{\text {ghi }}$ & $-1,2$ & $148,8^{\text {cde }}$ & $-4,1$ & $1,6^{\mathrm{cde}}$ & $-0,2$ \\
\hline CV (\%) & 4,5 & & 4,6 & & 7,9 & \\
\hline $\mathrm{F}(\mathrm{t})$ & $16,0^{* *}$ & & $9,0^{* *}$ & & $12,3^{* *}$ & \\
\hline
\end{tabular}

Trong cùng một cột, các trị số có cùng ký tự đi kèm khác biệt không có ý nghĩa về mặt thống kê, ** khác biệt rất có ý nghĩa (mức $\alpha=0,01$ ).

Theo Bảng 4, các tổ hợp lai với tester T1 đạt năng suất thực thu là từ 26,0 đến 43,1 tấn/ha, trong đó tổ hợp lai L28/T1 có năng suất thực thu cao nhất là 43,1 tấn/ha so với các tổ hợp lai còn lại. Đối với tester T2 các tổ hợp lai đạt năng suất thực thu là từ 27,0 đến 41,3 tấn/ha, trong đó tổ hợp lai L71/T2 có năng suất thực thu cao nhất là 41,3 tấn/ha. Kết quả này cũng phù hợp với mục tiêu chọn giống dưa leo cho khu vực Đông Nam Bộ, theo Tran \& ctv. (2012), chọn các tổ hợp lai giống dưa leo có triển vọng có năng suất thực thu từ 35 đến 40 tấn/ha.

Phân tích khả năng kết hợp chung về chỉ tiêu số khối lượng quả với vật liệu thử $\mathrm{T} 1$ và $\mathrm{T} 2$, có 7 dòng có khả năng kết hợp chung cao, giá trị KNKHC từ $(+) 0,3$ đến $(+) 5,4$ và 8 dòng không có khả năng kết hợp chung, giá trị KNKHC âm (-) (Bảng 4).

Qua quá trình theo dõi nhận thấy các tổ hợp lai dưa leo bị nhiễm nhẹ bệnh giả sương mai ở mức dưới $20 \%$ diện tích lá nhiễm bệnh, không bị nhiễm bệnh phấn trắng và vi rút. 
Bảng 4. Khả năng kết hợp chung (KNKHC) về chỉ tiêu năng suất thực thu của 15 dòng dưa leo thế hệ $\mathrm{I}_{5}$

\begin{tabular}{cccccc}
\hline \multirow{2}{*}{ Dòng dưa leo } & \multicolumn{4}{c}{ Năng suất thực thu của các tổ hợp lai dưa leo (tấn/ha) } \\
\cline { 2 - 6 } & T1 & KNKHC-T1 & T2 & KNKHC-T2 & KNKHC-TB \\
\hline L3 & $40,0^{\mathrm{b}}$ & 6,4 & $38,0^{\mathrm{cd}}$ & 2,7 & 4,6 \\
L9 & $37,9^{\mathrm{b}}$ & 4,3 & $36,1^{\mathrm{e}}$ & 0,8 & 2,6 \\
L21 & $40,7^{\mathrm{b}}$ & 7,1 & $37,2^{\text {de }}$ & 1,9 & 4,5 \\
L22 & $32,2^{\mathrm{d}}$ & $-1,4$ & $28,6^{\mathrm{g}}$ & $-6,7$ & $-4,1$ \\
L28 & $43,1^{\mathrm{a}}$ & 9,6 & $36,4^{\mathrm{e}}$ & 11 & 5,4 \\
L32 & $26,3^{\text {fg }}$ & $-7,3$ & $38,4^{\mathrm{c}}$ & 3,1 & $-2,1$ \\
L33 & $38,0^{\mathrm{c}}$ & 4,4 & $27^{\mathrm{h}}$ & $-8,3$ & $-2,0$ \\
L36 & $27,8^{\mathrm{f}}$ & $-5,8$ & $39,2 \mathrm{bc}$ & 3,9 & $-1,0$ \\
L39 & $26,5^{\text {fg }}$ & $-7,1$ & $29,1^{\mathrm{g}}$ & $-6,2$ & $-6,7$ \\
L61 & $40,7^{\mathrm{b}}$ & 7,1 & $38,7^{\mathrm{bc}}$ & 3,4 & 5,3 \\
L63 & $26,0 \mathrm{~g}$ & $-7,5$ & $39,8^{\mathrm{b}}$ & 4,5 & $-1,5$ \\
L69 & $26,6 f^{\mathrm{g}}$ & $-7,0$ & $29,9^{\text {fg }}$ & $-5,5$ & $-6,3$ \\
L71 & $37,3^{\mathrm{b}}$ & 3,8 & $41,3^{\mathrm{a}}$ & 6,0 & 4,9 \\
L74 & $30,3^{\mathrm{e}}$ & $-3,3$ & $39,2^{\mathrm{bc}}$ & 3,8 & 0,3 \\
L78 & $30,2^{\mathrm{e}}$ & $-3,4$ & $30,8^{\mathrm{f}}$ & $-4,5$ & $-4,0$ \\
\hline CV (\%) & 1,8 & \multicolumn{5}{c}{1,5} & \\
F(t) & $296,3^{* *}$ & $239,4^{* *}$ & & \\
\hline
\end{tabular}

Trong cùng một cột, các trị số có cùng ký tự đi kèm khác biệt không có ý nghĩa về mặt thống kê,

** khác biệt rất có ý nghĩa (mức $\alpha=0,01$ ).

Qua kết quả đánh giá về khả năng kết hợp chung của 15 dòng dưa leo thế hệ $\mathrm{I}_{5}$ tóm lại như sau: dòng dưa leo có khả năng kết hợp chung dòng tester T1 gồm 7 dòng (L3, L9, L21, L28, L33, L61 và L71), có khả năng kết hợp chung dòng tester T2 gồm 10 dòng (L3, L9, L21, L28, L32, L36, L61, L63, L71 và L74) về các chỉ tiêu tổng số quả trên cây, khối lượng quả, khối lượng quả/cây, năng suất thực thu.

Theo Kumari \& Kumar (2017), phân tích khả năng kết hợp chung là một trong những biện pháp để đưa ra các ước tính về việc kết hợp các hiệu ứng và hỗ trợ chọn lọc các dòng bố mẹ mong muốn. Nghiên cứu trên 6 dòng dưa leo và 2 dòng thử, dòng bố mẹ có khả năng kết hợp chung cao về năng suất được sử dụng thực hiện lai tạo giống F1 đạt ưu thế lai vượt trội ở các con lai.

\section{Kết Luận}

Từ kết quả đánh giá khả năng kết hợp chung của 15 dòng dưa leo thế hệ I5 với 2 dòng tester T1 và $\mathrm{T} 2$, qua các chỉ tiêu về năng suất chọn được 07 dòng có khả năng kết hợp chung cao với 2 dòng tester $\mathrm{T} 1$ và $\mathrm{T} 2$ gồm $\mathrm{L} 3, \mathrm{~L} 9, \mathrm{~L} 21, \mathrm{~L} 28, \mathrm{~L} 33, \mathrm{~L} 61$ và L71. 07 dòng dưa leo được chọn, đáp ứng được yêu mục tiêu của nghiên cứu và được sử dụng làm dòng bố mẹ trong thí nghiệm đánh giá khả năng kết hợp riêng, chọn tạo giống dưa leo F1. Các tổ hợp lai được tạo thành từ 07 dòng được chọn với 2 dòng tester T1 và T2 có sự sinh trưởng mạnh, các yế tố cấu thành năng suất và năng suất cao và ít bị ảnh hưởng bệnh hại. Trong đó có 2 tổ hợp lai có năng suất cao có ý nghĩa khác biệt so với các tổ hợp lai khác là L28/T1 (43,1 tấn/ha) và $\mathrm{L} 71 / \mathrm{T} 2$ (41,3 tấn/ha).

\section{Tài Liệu Tham Khảo (References)}

AHRD (High-tech Agricultural Reasearch and Development Center). (2016). The Process of bitter gourd (Momordica charantia L.) cultivation. Ho Chi Minh City, Vietnam: AHRD.

AHRD (High-tech Agricultural Reasearch and Development Center). (2015). Growing plants in substratesin a net house. Ho Chi Minh City, Vietnam: AHRD.

FAOSTAT (Food and Agriculture Organization Corporate Statistical Database). (2020). Crops data. Retrieved August 10, 2020, from http://www.fao.org/faostat/en/\#data/QC.

Hoang, H. D., Nguyen, P. T. B., To, T. T. T., Tran, L. V., Le, M. T. T., Huynh, T. Q., \& Thai, B. T., (2019). Selection of source materials and cucumber varieties (Cucumis sativus L.) suitable to the Southeast region of Vietnam ( $3^{\text {rd }}$ year). Ho Chi Minh City, Vietnam: Research and Development Center for Hi-tech Agriculture. 
Hoang, T. P., \& Truong, T. B. P. (2008). Genetic basis of plant breeding. Hue, Vietnam: Hue University Publishing House.

Kumari, R., \& Kumar, S. (2017). Combining ability studies for yield and yield contributing traitsin $F_{1}$ and $F_{2}$ generations of cucumber (Cucumis sativus L.). Indian Journal of Ecology 44(6), 852-855.

MARD (Ministry of Agriculture and Rural Development). (2012). QCVN 01-87:2012/BNNPTNT dated on June 19, 2012. National technical regulation on testing for value of cultivation and use of cucumber varieties. Retrieved February 04, 2021, from https://vdocuments.net/qcvn-01-87-2012bnnptntdoc.html.

Ngo, H. T. (2011). Selection of source materials and cucumber varieties (Cucumis sativus L.) for processing (Unpublished doctoral dissertation). Hanoi University of Agriculture, Ha Noi, Vietnam.
Phan, K. T. (2006). Plant breeding curriculum. Ha Noi, Vietnam: Agricultural Publishing House.

Tatlioglu, T. (1993). Cucumber: Cucumis sativus L. In Kalloo, G., \& Bergh, B. O. (Eds). Genetic improvement of vegetable crops (197-234). Oxford, UK: Pergamon.

Tran, C. K., Le, S. T., Nguyen ,V. N., Huynh, L. T. P., Duong, T. K., Pham, L. M., Dao, T. X., \& Nguyen, C. M. (2012). Results on breeding of tomato, cucumber, okra and eggplant. VAAS 2012 First National Conference on Plant Science (499-506). Hanoi: Vietnam Academy of Agricultural Sciences. 